\title{
EFEITO DA CALAGEM NA RELAÇÃO ENTRE SOLO E ÁGUA(1)
}

\author{
Edson Campanhola Bortoluzzi ${ }^{(2)}$, Leandro Garbozza ${ }^{(3)}$, Caroline Guareschi $^{(4)}$ \& Danilo \\ dos Santos Rheinheimer ${ }^{(5)}$
}

\begin{abstract}
RESUMO
Mudanças nas propriedades físicas do solo, principalmente aquelas relacionadas com a retenção de água e agregação, podem estar associadas às variações na eletroquímica do solo. Estudar o efeito da calagem sobre a relação entre solo e água constituiu o objetivo deste trabalho. Em 1994, um experimento foi instalado na área experimental do Departamento de Solos da Universidade Federal de Santa Maria, em um Argissolo Acinzentado distrófico plíntico. Os tratamentos consistiram na aplicação superficial e incorporação de calcário nas doses de zero, 2+2, 8,5 e $17 \mathrm{Mg} \mathrm{ha}^{-1}$. Em 2006, doze anos após a aplicação de calcário, foram coletadas amostras de solo com estrutura preservada e em triplicata nas camadas de 0-5 cm e 5-10 cm. As amostras foram caracterizadas quanto a algumas propriedades químicas. As propriedades físicas avaliadas foram: i) velocidade de umectação do solo, estimada em um dispositivo de ascensão capilar de água; ii) umidade gravimétrica do solo, medida ao final do teste de ascensão capilar de água, e iii) densidade do solo, estimada pela razão entre a massa e o volume de torrões de solo seco. A velocidade de umectação foi mais intensa nos solos que receberam as maiores doses de calcário. A umidade gravimétrica aumentou linearmente com as doses de calcário, enquanto a densidade do solo não foi alterada. Houve incremento de água no solo entre 5,1 e $6,2 \mathrm{mg} \mathrm{g}^{-1}$ para cada tonelada de corretivo aplicada. Podese concluir que, após 12 anos da calagem, as formas de aplicação de calcário não tiveram influência sobre a densidade do solo e sobre a retenção de água; a calagem alterou a relação entre solo e água, tornando o solo mais hidrófilo com o aumento das doses de calcário, independentemente da forma de aplicação do corretivo.
\end{abstract}

Termos de indexação: capacidade de troca de cátions, hidratação de cátions, cargas negativas permanentes, ascensão capilar, retenção de água.

\footnotetext{
(1) Trabalho apresentado na XVI Mostra de Iniciação Científica da UPF. Trabalho apresentado no XXXI Congresso Brasileiro de Ciência do Solo, 2007 (Gramado, RS).

(2) Professor Adjunto II da Faculdade de Agronomia e Medicina Veterinária - FAMV da Universidade de Passo Fundo - UPF, Caixa Postal 611, Bairro São José, Passo Fundo (RS), Brasil, CEP 99001-970. E-mail: edsonb@upf.br

(3) Acadêmico do curso de Agronomia da UPF. Bolsista voluntário de iniciação científica. E-mail: garbozza_81@yahoo.com.br

(4) Acadêmica do curso de Química da UPF. Bolsista de iniciação científica da FAPERGS. E-mail: karol_quim@yahoo.com.br.

(5) Professor Adjunto do Departamento de Solos da Universidade Federal de Santa Maria (UFSM). Bolsista de produtividade em pesquisa CNPq. E-mail: danilor@smail.ufsm.br
} 


\title{
SUMMARY: EFFECTS OF LIMING ON THE RELATIONSHIP BETWEEN SOIL AND WATER
}

\begin{abstract}
Changes in the physical properties of soil, mainly related to water retention and soil aggregation, may be linked to changes in electrochemical soil properties. The purpose of this study was to determine the effects of liming on the relationship between soil and water. In 1994, an experiment was installed in the experimental area of the Departamento de Solos da Universidade Federal de Santa Maria, in a dystrophic plinthic Gray Acrisol. Treatments consisted in surface liming and lime incorporation at $0,2+2,8.5$, and $17 \mathrm{Mg} \mathrm{ha}^{-1}$. In 2006, 12 years after lime application, undisturbed soil samples were collected in triplicate from the layers $0-5$ and $5-10 \mathrm{~cm}$. The chemical properties of the samples were characterized. The following physical properties were evaluated i) speed of soil wettability, estimated using a capillary rise device, ii) gravimetric soil moisture, measured at the end of the capillary rise trials, iii) bulk density, estimated by the ratio between the dry mass and volume of soil clods. The wettability speed was higher in soils treated with higher limestone doses, while bulk density was not affected. There was an increase of soil water contents of around 5,1 and 6,2 $\mathrm{mg} \mathrm{g}^{-1}$ for each tonne of lime applied. The conclusion was drawn that the lime application forms had no influence on bulk density and soil water retention; liming influences the soil - water relationship, making the soil more hydrophilic with increasing doses of lime, regardless of the form of lime application.
\end{abstract}

Index terms: cation exchange capacity, cation hidratation, negative permanent charges, capillary rise, water retention.

\section{INTRODUÇÃO}

A calagem é prática importante em solos cujas propriedades químicas são regidas por cargas elétricas variáveis ou dependentes do $\mathrm{pH}$. O cultivo de solos sob campo nativo, principalmente aqueles do Rio Grande do Sul, exigiu melhorias nas propriedades químicas, sem que a estrutura do solo fosse alterada. Assim, o cultivo em sistema plantio direto e a calagem realizada na superfície do solo sem incorporação do corretivo contribuíram para contemplar esse objetivo. Melhorias nas propriedades químicas foram constatadas em superfície (Rheinheimer et al., 2000a), concomitantemente à manutenção de boas características físicas do solo, como a estabilidade da estrutura (Costa et al., 2004).

Estudos que visam relacionar os efeitos da calagem sobre propriedades físicas do solo são de difícil diagnóstico, principalmente em experimentos recentes. Isso por que tais efeitos ocorrem de forma indireta, pela ação do implemento na incorporação do corretivo, ou, ainda, pelo incremento na atividade microbiana ou do sistema radicular das culturas (Griève et al., 2005). Contudo, resultados obtidos em experimentos de longa duração evidenciam alterações na retenção de água no solo (Tessier, 2001; Bortoluzzi \& Tessier, 2002) e na estabilidade da estrutura de acordo com a magnitude da CTC e da espécie química predominante nos sítios de troca (Pernes-Debuyser \& Tessier, 2004; Bortoluzzi et al., 2005).

O caráter hidrófilo-hidrófobo do solo pode variar em função da eletroquímica do solo (Prost et al., 1998; Chenu et al., 2001; Pernes-Debuyser \& Tessier, 2004). É notório que superfícies sólidas têm afinidades diferenciadas pelas moléculas de água, sendo estimadas pelo ângulo de contato formado entre o menisco da gota de água sobre a superfície (Jouanny et al., 1992). Superfícies hidrófobas apresentam um ângulo de contato grande, superior a $90^{\circ}$, enquanto ângulos pequenos denotam uma superfície hidrófila. Goebel et al. (2005) verificaram que, quanto maior o ângulo de contato entre a água e o solo, maior é a estabilidade de agregados de solo. Peltier (2001), ao estudar solos ácidos da Europa, verificou que a calagem com cal virgem propiciou uma melhor aeração e retenção de água nesses solos.

Nesse contexto, estima-se que a calagem interfira não só nas forças de coesão e repulsão entre as partículas do solo, mas também na afinidade da fase sólida e as moléculas de água, por alterar a eletroquímica do solo (Prost et al., 1998). Por conseqüência, propriedades físicas macroscópicas, como a retenção de água e a estabilidade dos agregados, podem ser alteradas pela prática da calagem.

O trabalho foi realizado com o intuito de estudar os efeitos da calagem sobre as propriedades físicas do solo, em especial aquelas relacionadas com a retenção de água e velocidade de umectação de um Argissolo do Sul do Brasil.

\section{MATERIAL E MÉTODOS}

\section{Localização e característica da área do experimento}

Um experimento, destinado ao estudo de formas de aplicação e doses de calcário, localizado no campo experimental do Departamento de Solos da 
Universidade Federal de Santa Maria (29 43’ 04" S e $53^{\circ} 42^{\prime} 06^{\prime}$ W), Rio Grande do Sul, foi utilizado para o presente trabalho. O solo é classificado como um Argissolo Acinzentado distrófico plíntico (Embrapa, 2006), com textura média e substrato formado por sedimentos aluviais recentes.

A área do experimento, com relevo suave ondulado, foi mantida sob pastagem natural até 1994, quando foi dessecada quimicamente e parcelas de $12 \times 12 \mathrm{~m}$ foram demarcadas. A análise química do solo na camada de 0 $-20 \mathrm{~cm}$, por ocasião da instalação do experimento, foi realizada segundo Tedesco et al. (1995). Nela, foram avaliados os seguintes atributos: teores de argila, 190 $\mathrm{g} \mathrm{kg}^{-1}$; silte, $260 \mathrm{~g} \mathrm{~kg}^{-1}$; areia, $550 \mathrm{~g} \mathrm{~kg}^{-1} ; \mathrm{pH}$ em água, 4,4; teor de matéria orgânica, $37 \mathrm{~g} \mathrm{dm}^{-3}$; Al trocável, $3,30 \mathrm{cmol}_{\mathrm{c}} \mathrm{dm}^{-3} ; \mathrm{Ca}+\mathrm{Mg}$ trocáveis, $3,7 \mathrm{cmol}_{\mathrm{c}} \mathrm{dm}^{-3} ; \mathrm{P}$ disponível, $2,3 \mathrm{mg} \mathrm{dm}^{-3}$ e $\mathrm{K}$ trocável, $54 \mathrm{mg} \mathrm{dm}^{-3}$. O pH SMP foi de 4,5, correspondendo à necessidade de 17 $\mathrm{Mg} \mathrm{ha}^{-1}$ de calcário com poder relativo de neutralização total de $100 \%$ (CQFSRS/SC, 2004).

Os tratamentos consistiram na aplicação a lanço de diferentes doses de corretivo da acidez do solo. Em metade das parcelas, o calcário foi incorporado até 20 cm por meio de uma lavração e duas passagens de grade. As doses de calcário dolomítico foram: zero, $2,0,8,5$ e $17,0 \mathrm{Mg} \mathrm{ha}^{-1}$, sendo a dose de $2 \mathrm{Mg} \mathrm{ha}^{-1}$ reaplicada em superfície dois anos após a primeira aplicação $\left(2+2 \mathrm{Mg} \mathrm{ha}^{-1}\right)$. A área, desde o início do experimento, foi cultivada em sistema plantio direto, utilizando-se a rotação de culturas tradicional da região, com milho/soja/azevém/trevo. Detalhes do experimento podem ser encontrados em Kaminski et al. (2005).

\section{Coleta e análises das amostras}

Em 2006, foram coletadas, com auxílio de um cilindro de PVC com diâmetro de $10 \mathrm{~cm}$, amostras de solo com estrutura preservada das camadas de 0-5 cm e 5-10 cm. A terra fina seca ao ar (TFSA) foi submetida, no laboratório de Química do Solo da Universidade Federal de Santa Maria, à caracterização dos atributos químicos, como $\mathrm{pH}$ em água e SMP, cátions trocáveis- $\mathrm{Ca}^{2+}, \mathrm{Mg}^{2+}, \mathrm{K}^{+}, \mathrm{Al}^{3+}$, soma de bases-SB, capacidade de troca de cátionsCTC, efetiva-CTC $\mathrm{E}$ e a pH 7-CTC 7 , segundo Tedesco et al. (1995). (Quadro 1).

Quadro 1. Atributos químicos de um Argissolo nas camadas de 0-5 cm e 5-10 cm sob sistema plantio direto e aplicação superficial e incorporação de doses de calcário

\begin{tabular}{|c|c|c|c|c|c|c|c|c|c|c|c|c|c|}
\hline \multirow{2}{*}{ Doses } & \multirow{2}{*}{ Camada } & \multicolumn{2}{|c|}{$\mathbf{p H}$} & \multicolumn{5}{|c|}{ Cátions trocáveis } & \multirow{2}{*}{$\mathrm{CTC}_{\mathrm{E}}$} & \multirow{2}{*}{$\mathrm{CTC}_{7}$} & \multirow{2}{*}{ SB } & \multicolumn{2}{|c|}{ Saturação por } \\
\hline & & água & SMP & $\mathbf{K}^{+}$ & $\mathbf{C a}^{2+}$ & $\mathbf{M g}^{2+}$ & $\mathrm{Al}^{3+}$ & $\mathrm{H}+\mathrm{Al}$ & & & & $\mathrm{Al}$ & Bases \\
\hline $\mathrm{Mg} \mathrm{ha}^{-1}$ & $\mathrm{~cm}$ & $1: 1$ & $1: 1: 0,5$ & & & 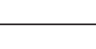 & \multicolumn{2}{|c|}{$-\mathrm{cmol}_{\mathrm{c}} \mathrm{dm}^{-3}$} & & & & \multicolumn{2}{|c|}{$\%$} \\
\hline \multicolumn{14}{|c|}{ Superficial } \\
\hline \multirow[t]{2}{*}{0,0} & $0-5$ & 4,2 & 4,4 & 0,34 & 4,1 & 1,4 & 3,3 & 26,7 & 9,1 & 32,5 & 5,8 & 36,3 & 18,4 \\
\hline & $5-10$ & 4,3 & 4,3 & 0,16 & 2,9 & 0,8 & 5,0 & 31,5 & 8,9 & 35,3 & 3,9 & 56,2 & 11,8 \\
\hline \multirow[t]{2}{*}{$2+2$} & $0-5$ & 5,1 & 5,5 & 0,34 & 8,0 & 3,6 & 0,3 & 8,2 & 12,3 & 20,1 & 12,0 & 2,6 & 59,3 \\
\hline & $5-10$ & 4,7 & 4,8 & 0,14 & 3,8 & 1,9 & 2,5 & 18,9 & 8,3 & 24,8 & 5,8 & 28,8 & 24,8 \\
\hline \multirow[t]{2}{*}{8,5} & $0-5$ & 4,9 & 5,4 & 0,32 & 6,7 & 3,1 & 0,6 & 9,1 & 10,7 & 19,2 & 10,1 & 6,3 & 51,7 \\
\hline & $5-10$ & 4,7 & 5,1 & 0,19 & 5,0 & 2,4 & 1,9 & 12,4 & 9,4 & 19,9 & 7,5 & 19,8 & 38,5 \\
\hline \multirow[t]{2}{*}{17} & $0-5$ & 5,7 & 6,0 & 0,29 & 10,3 & 4,1 & 0,0 & 4,7 & 14,6 & 19,3 & 14,6 & 0,1 & 76,5 \\
\hline & $5-10$ & 5,3 & 5,6 & 0,15 & 6,8 & 3,6 & 1,3 & 7,6 & 11,9 & 18,2 & 10,6 & 12,2 & 58,2 \\
\hline \multicolumn{14}{|c|}{ Incorporação } \\
\hline \multirow[t]{2}{*}{0,0} & $0-5$ & 4,4 & 4,7 & 0,37 & 4,2 & 1,7 & 2,9 & 19,7 & 9,2 & 26,0 & 6,3 & 32,4 & 24,0 \\
\hline & $5-10$ & 4,4 & 4,5 & 0,14 & 3,0 & 1,1 & 4,3 & 25,1 & 8,6 & 29,4 & 4,3 & 49,8 & 14,9 \\
\hline \multirow[t]{2}{*}{$2+2$} & $0-5$ & 5,2 & 5,6 & 0,41 & 7,2 & 3,5 & 0,3 & 7,2 & 11,4 & 18,3 & 11,1 & 2,9 & 61,3 \\
\hline & $5-10$ & 4,7 & 5,0 & 0,17 & 4,6 & 2,2 & 2,0 & 14,0 & 9,0 & 20,9 & 6,9 & 22,4 & 33,5 \\
\hline \multirow[t]{2}{*}{8,5} & $0-5$ & 4,7 & 5,1 & 0,36 & 6,1 & 2,7 & 1,1 & 12,2 & 10,3 & 21,3 & 9,2 & 10,5 & 43,8 \\
\hline & $5-10$ & 4,8 & 5,1 & 0,18 & 5,0 & 2,2 & 2,0 & 13,6 & 9,3 & 21,0 & 7,4 & 21,0 & 37,8 \\
\hline \multirow[t]{2}{*}{17,0} & $0-5$ & 5,2 & 5,7 & 0,30 & 7,5 & 3,3 & 0,3 & 6,2 & 11,4 & 17,3 & 11,1 & 2,2 & 64,4 \\
\hline & $5-10$ & 5,4 & 5,7 & 0,15 & 7,5 & 3,8 & 0,3 & 6,7 & 11,7 & 18,1 & 11,4 & 2,9 & 63,5 \\
\hline
\end{tabular}

$\mathrm{CTC}_{\mathrm{E}}=$ capacidade de troca de cátions efetiva; $\mathrm{CTC}_{7}=$ capacidade de troca de cátions a pH 7,0; $\mathrm{SB}=$ soma de bases $\left(\mathrm{Ca}^{2+}+\mathrm{Mg}^{2+}+\mathrm{K}^{+}\right)$. 
Nas amostras com estrutura preservada, foram separados torrões de tamanho entre 3 e $6 \mathrm{~cm}^{3}$, pesados quando úmidos e sob imersão em querosene e secos a $105{ }^{\circ} \mathrm{C}$. Pôde-se, dessa forma, calcular o volume dos torrões, a densidade aparente do solo (Ds) e a umidade gravimétrica (Ug), segundo Monnier et al. (1973).

A velocidade de umectação foi determinada, em triplicata, sobre alíquotas de $40 \mathrm{~g}$ de TFSA passadas em peneira de malha $1 \mathrm{~mm}$. A determinação seguiu o método descrito por Michel et al. (2001). Em resumo, as amostras foram colocadas no interior de um tubo de PVC, com $10 \mathrm{~cm}$ de altura e $2,5 \mathrm{~cm}$ de diâmetro, com uma tampa porosa e rígida servindo de suporte e permitindo a ascensão livre da água pela amostra. Um conjunto de dois recipientes, conectados por vasos comunicantes, foi mantido em equilíbrio de massas com água deionizada sobre o prato da balança analítica e um suporte regulável. Instalado o tubo de PCV com a amostra dentro do recipiente sobre a balança, a massa do conjunto foi determinada em intervalos de tempo de $15 \mathrm{~s}$, até alcançar a máxima retenção de água. Tem-se como premissa básica da experiência a condição de homogeneidade quanto à porosidade da amostra dentro do tubo de PVC. Assim, a entrada de água na amostra é regida unicamente pela ascensão capilar, evidenciando forças de atração das partículas sólidas com a água e o caráter hidrófilo da amostra.

Conhecendo a massa inicial, durante o processo de ascensão capilar e ao final, é possível determinar a retenção de água da amostra assim como a velocidade de umectação. A umidade gravimétrica ao final do processo indica a máxima retenção de água da amostra (Ug) e a velocidade de umectação indica a afinidade da fração sólida pela água ou o caráter hidrófilo/hidrófobo do solo que pode ser discutido à luz da eletroquímica do solo (Pernes-Debuyser \& Tessier, 2004).

Gráficos que relacionam a umidade gravimétrica da amostra a cada unidade de tempo foram construídos, permitindo determinar a velocidade de umectação pela inclinação de segmentos de reta. A correlação de Pearson foi efetuada entre os dados químicos e físicos. A Anova foi utilizada unicamente para as propriedades físicas de cada camada de solo, considerando um experimento em blocos ao acaso com parcelas subdivididas em faixas e três repetições. As parcelas principais constituíram-se nas doses de corretivo e as subparcelas nas formas de aplicação de calcário. Regressões foram feitas para os dados quantitativos quando indicadas pela Anova.

\section{RESULTADOS E DISCUSSÃO}

Os atributos químicos do solo para a acamada de 0-5 cm de profundidade (Quadro 1) mostram que o $\mathrm{Al}$ trocável apresentou valores próximos a zero, nas doses de 8,5 e $17 \mathrm{Mg} \mathrm{ha}^{-1}$, quando o calcário foi aplicado em superfície, e na doses de $17 \mathrm{Mg} \mathrm{ha}^{-1}$, quando incorporado. A maior dissociação de $\mathrm{Ca}$ e $\mathrm{Mg}$ nas maiores doses de calcário permitiu o aumento da saturação da CTC por estes cátions. Esse processo é mais intenso na camada mais superficial e nas maiores doses do corretivo aplicado em superfície, como também verificado por Kaminski et al. (2005). No entanto, apenas a dose de $17 \mathrm{Mg} \mathrm{ha}^{-1}$ manteve a saturação por bases próxima a $65 \%$, nas camadas de 0-5 cm e 5-10 cm. Em geral, o aumento nas doses de corretivo da acidez do solo aumentou os valores de $\mathrm{pH}, \mathrm{Ca}^{2+}, \mathrm{Mg}^{2+}, \mathrm{CTC}_{\mathrm{E}}$ e $\mathrm{CTC}_{7}$, principalmente quando aplicado em superfície. Os valores de $\mathrm{pH}$ e $\mathrm{Al}^{3+}$ mantiveram-se inalterados em profundidade, em relação à dose zero, considerando principalmente, a baixa solubilidade do calcário e a frente de alcalinização situada nas camadas superficiais de solo (Rheinheimer et al., 2000b). Esses resultados reforçam os apresentados por Kaminski et al. (2005), servindo aqui para corroborar que, após 12 anos do início do experimento, ainda foram visualizados os efeitos da calagem sobre a eletroquímica do solo. Espera-se que esse período minimize os efeitos indiretos da forma de aplicação de calcário e evidencie a condição da eletroquímica do solo como atuante nas mudanças das propriedades físicas avaliadas.

Verificou-se que a densidade do solo não se correlaciou significativamente com as propriedades químicas avaliadas (Quadro 2). Entretanto, correlações negativas de $r=-0,47$ e $r=-0,42$ foram evidenciadas entre a umidade gravimétrica e os valores de $\mathrm{H}+\mathrm{Al}$ e $\mathrm{CTC}_{7}$, respectivamente. Isso denota que há uma tendência de quanto maior a acidez do solo, menor será a umidade gravimétrica. Nessa lógica, permite-se inferir que, quando há aumento nos valores da soma de bases (Ca e Mg) decorrentes da calagem, a umidade gravimétrica do solo também tende a aumentar.

Quadro 2. Coeficiente de correlações de Pearson entre diferentes variáveis químicas e físicas avaliadas

\begin{tabular}{cccccccc}
\hline & $\mathbf{U g}$ & $\mathbf{p H ~ H} \mathbf{H}_{2} \mathbf{O}$ & $\mathbf{p H ~ S M P}$ & $\mathbf{S B}$ & $\mathbf{H}+\mathbf{A l}$ & $\mathbf{C T C}_{\mathbf{E}}$ & $\mathbf{C T C}_{7}$ \\
\hline $\mathrm{Ds}^{(1)}$ & $0,39^{\mathrm{ns}}$ & $0,11^{\mathrm{ns}}$ & $0,09^{\mathrm{ns}}$ & $0,01^{\mathrm{ns}}$ & $-0,13^{\mathrm{ns}}$ & $-0,05^{\mathrm{ns}}$ & $-0,16^{\mathrm{ns}}$ \\
$\mathrm{Ug}^{(2)}$ & 1,00 & $0,50^{*}$ & $0,48^{*}$ & $0,40^{*}$ & $-0,47^{*}$ & 0,32 & $-0,42^{*}$ \\
\hline
\end{tabular}

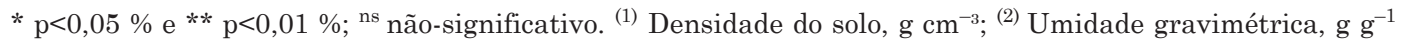


Quadro 3. Densidade de um Argissolo, nas camadas de 0-5 cm e 5-10 cm, após 12 anos da aplicação superficial e incorporação de doses de calcário

\begin{tabular}{|c|c|c|c|c|c|}
\hline \multirow{2}{*}{$\begin{array}{l}\text { Formas de } \\
\text { aplicação }\end{array}$} & \multicolumn{4}{|c|}{ Dose de calcário, $\mathbf{M g ~ h a ~}^{-1}$} & \multirow[b]{2}{*}{ Média } \\
\hline & Zero & $2+2$ & 8,5 & 17 & \\
\hline \multicolumn{6}{|c|}{ Densidade do solo, $\mathrm{g} \mathrm{cm}^{-3}$} \\
\hline \multicolumn{6}{|c|}{$0-5 \mathrm{~cm}$} \\
\hline Superficial & 1,64 & 1,63 & 1,71 & 1,72 & $1,68^{\text {ns }}$ \\
\hline Incorporação & 1,64 & 1,57 & 1,69 & 1,64 & 1,64 \\
\hline Média & $1,64^{\mathrm{ns}}$ & 1,60 & 1,70 & 1,68 & \\
\hline \multicolumn{6}{|c|}{$5-10 \mathrm{~cm}$} \\
\hline Superficial & 1,72 & 1,77 & 1,75 & 1,79 & $1,76^{\mathrm{ns}}$ \\
\hline Incorporação & 1,69 & 1,73 & 1,68 & 1,80 & 1,73 \\
\hline Média & $1,70^{* *}$ & 1,75 & 1,72 & 1,79 & \\
\hline
\end{tabular}

** $\mathrm{p}<0,01 \%,{ }^{\text {ns }}$ não-significativo.

A densidade do solo na camada de 0-5 cm não variou significativamente de acordo com as doses e formas de aplicação do calcário (Quadro 3). Contudo, para a camada de $5-10 \mathrm{~cm}$, a densidade do solo pode ser explicada em $69 \%\left(R^{2}=0,69\right)$ pelas doses de calcário aplicadas, representada pela equação linear de regressão $\hat{y}=1,71+0,0047 x$. Apesar da significância estatística, há pouco significado agronômico para essa relação, visto que a densidade do solo é alta mesmo para solos arenosos, variando apenas na segunda casa decimal de $1,70 \mathrm{~g} \mathrm{~cm}^{-3}$ a $1,79 \mathrm{~g} \mathrm{~cm}^{-3}$. Observa-se que os valores obtidos para a densidade do solo foram similares para ambas as formas de aplicação de corretivo. Isso demonstra que o efeito da incorporação do calcário sobre a densidade do solo é minimizado com a adoção e manutenção por 12 anos do sistema plantio direto. Os resultados obtidos neste estudo discordam daqueles de Griève et al. (2005) que verificaram um incremento na porosidade do solo, logo uma diminuição da densidade do solo com a calagem. Isso é explicado, provavelmente, pelo pequeno intervalo de tempo decorrido entre a aplicação do corretivo e as avaliações realizadas.

As curvas de velocidade de umectação das amostras de solo de acordo com as doses caracterizam-se por hipérboles (Figura 1). Nelas, percebe-se uma rápida ascensão capilar da água no início do teste, estabilizando-se em seguida. Assim, a velocidade de umectação é maior nos primeiros minutos de contato da amostra com a água, o que denota maior afinidade sólido/água nessa primeira fase, explicada pelo potencial matricial das partículas (Prost et al., 1998). Já, na segunda fase, há uma estabilização e a velocidade em que a água ascende na amostra diminui. Percebe-se que a ascensão capilar de água no limite máximo de retenção de água do solo tende a ser nula.
Em geral, o teor de água no solo tendeu a se elevar mais rapidamente com o aumento das doses de corretivo (Figura 1). Para a camada de 0-5 cm, a velocidade de umectação do solo tendeu a ser mais intensa em amostras de solos que receberam as maiores doses de calcário, ou seja, doses de 8,5 e 17 Mg ha-1 (Figura 1a b). Entretanto, quando o calcário foi incorporado, as curvas de velocidade de umectação apresentaram-se em dois grupos. $\mathrm{O}$ primeiro, referente às doses de zero e $2+2 \mathrm{Mg} \mathrm{ha}^{-1}$, apresentou menor velocidade de umectação e o segundo, referente às doses de 8,5 e $17 \mathrm{Mg} \mathrm{ha}^{-1}$, apresentou maior velocidade de umectação. Isso se explica pelo fato de que as menores doses de calcário, doses inferiores à recomendada, tiveram contato com um volume grande de solo, sendo incapazes de alterar as propriedades químicas e, por conseqüência, as propriedades físicas associadas (Quadro 1).

Contudo, na camada de solo de 5-10 cm com aplicação de calcário em superfície, as curvas de velocidade de umectação tenderam a se aproximar, com exceção daquela que representa a dose de $17 \mathrm{Mg}$ $\mathrm{ha}^{-1}$ (Figura 1c, d). Isto se deveu ao efeito pronunciado sobre a eletroquímica do solo em profundidade, favorecido pelo longo tempo que o calcário teve para reagir no solo (Kaminski et al., 2000; Rheinheimer et al., 2000a; Caíres et al., 2000). Em ambas as formas de aplicação do corretivo, percebeu-se comportamento similar para as duas camadas estudadas (Figura 1). Vale ressaltar que, ao final do ensaio, a umidade gravimétrica tendeu a ser maior nas amostras que receberam as maiores doses de calcário (Figura 1, 2 e 3).

A variação da umidade gravimétrica do solo foi explicada em no mínimo $90 \%$ pelo acréscimo nas doses de calcário no solo, $R^{2}=0,97$ e 0,92 para as 


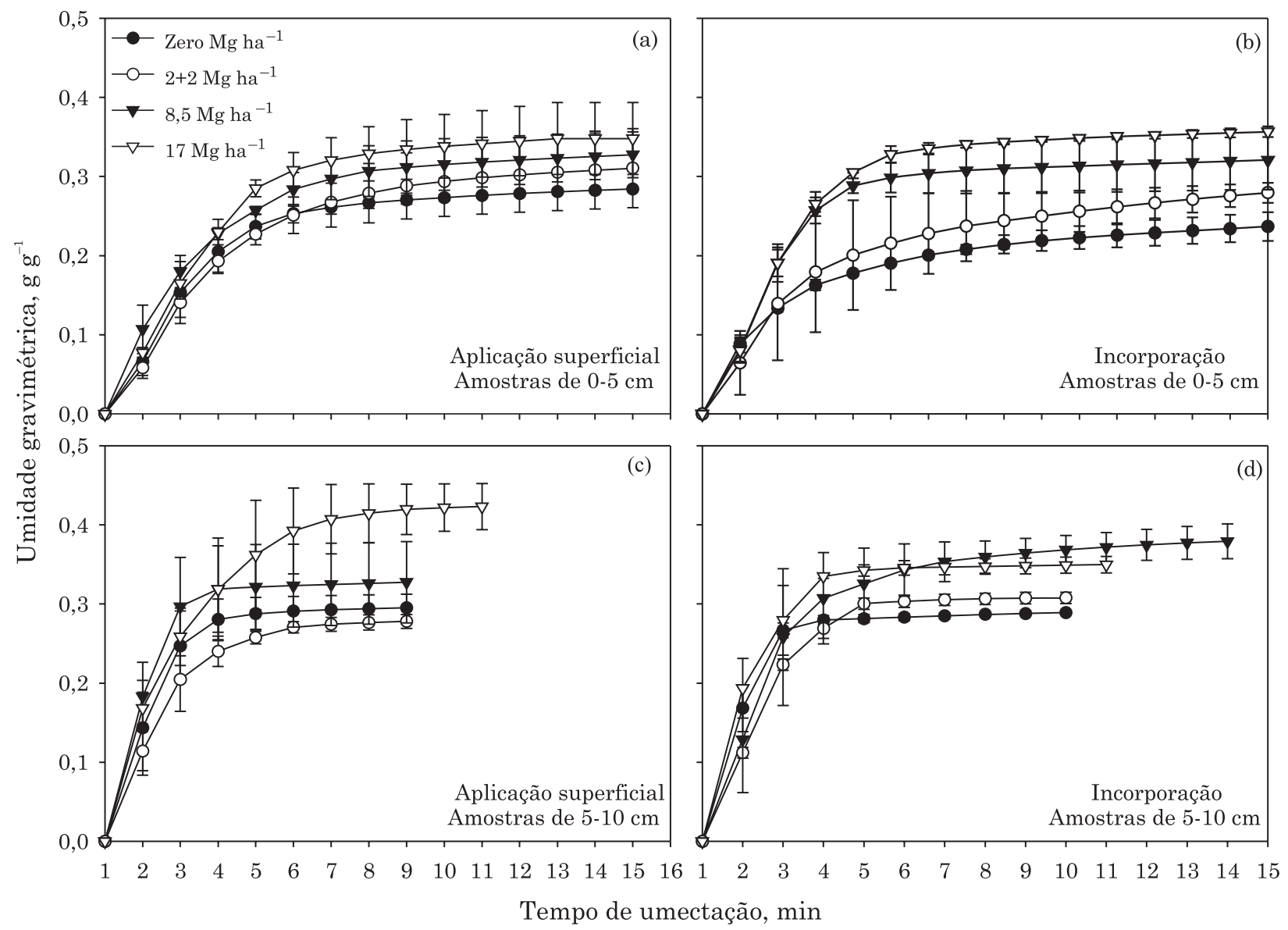

Figura 1. Velocidade de umectação de um Argissolo que recebeu em 1994 diferentes doses de calcário em duas formas de aplicação. Amostras da profundidade de 0-5 cm (a) superficial e (b) incorporado, e de 5$10 \mathrm{~cm}$, (c) superficial e (d) incorporado. As barras verticais representam o desvio-padrão de três repetições em relação à média.

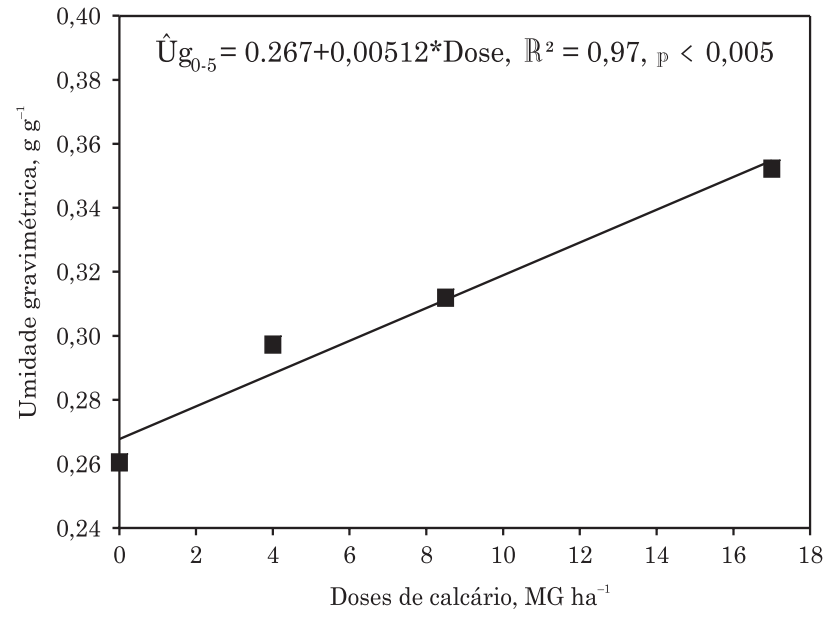

Figura 2. Relação entre a umidade gravimétrica na máxima retenção de água no ensaio de ascensão capilar de acordo com as doses de calcário aplicadas em um Argissolo, camada de 0-5 cm.

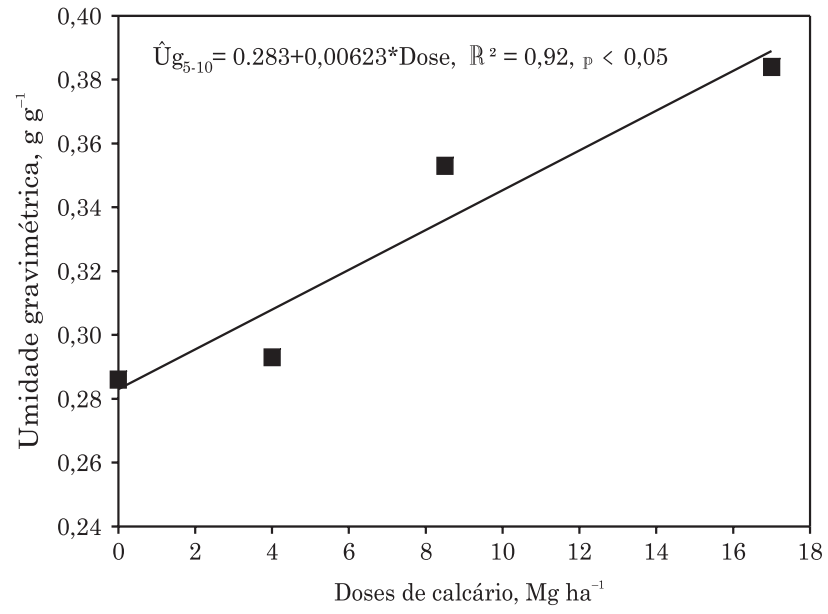

Figura 3. Relação entre a umidade gravimétrica na máxima retenção de água no ensaio de ascensão capilar de acordo com as doses de calcário aplicadas em um Argissolo, camada de 5-10 cm. 
camadas de 0-5 e 5-10 cm (Figura 2 e 3). Observouse que não houve interação entre as doses e as formas de aplicação do calcário, assim, a variação da umidade independeu da incorporação ou não do corretivo ao solo. Isso era esperado, pois indica que os efeitos indiretos da calagem, decorrentes do revolvimento do solo, sobre as propriedades físicas foram minimizados com o tempo.

Os valores de umidade gravimétrica do solo na camada de 0-5 cm partiram de $0,27 \mathrm{~g} \mathrm{~g}^{-1}$ na dose zero para $0,35 \mathrm{~g} \mathrm{~g}^{-1}$, na maior dose de corretivo aplicada, um aumento de cerca de $30 \%$ (Figura 2). Na camada de $5-10 \mathrm{~cm}$ (Figura 3), o incremento foi ainda maior, cerca de $35 \%$. Assim, para cada tonelada de calcário aplicada ao solo, houve um incremento na retenção de água na ordem de 5,1 e 6,2 $\mathrm{mg} \mathrm{g}^{-1}$ para as camadas de $0-5 \mathrm{~cm}$ e $5-10 \mathrm{~cm}$, respectivamente.

Pode-se inferir que o efeito na eletroquímica do solo decorrente da calagem, substituindo nos sítios de troca $\mathrm{O}^{+}$e $\mathrm{Al}^{3+}$ pelo $\mathrm{Ca}^{2+}$ e $\mathrm{Mg}^{2+}$, aumenta a afinidade da fase sólida do solo pela água, provavelmente por aumentar a espessura da dupla camada difusa e o potencial matricial das partículas de solo (Prost et al., 1998; Chenu et al., 2001; PernesDebuyser \& Tessier, 2004). Considerando que o ensaio partiu de uma condição de alto potencial de água, solo seco, espera-se que a mineralogia do solo e o estado das cargas elétricas atuem de forma preponderante na afinidade da fase sólida pela água (Prost et al., 1998; Bortoluzzi et al., 2005). Isso é válido para esse tipo de solo, visto que a sua mineralogia é composta por argilominerais do tipo $2: 1$, como vermiculita e esmectita com alta carga permanente e afinidade à água (Bortoluzzi et al., 2006, 2007, 2008). Assim, este estudo permite que inferências sejam feitas sobre a afinidade das partículas sólidas do solo pela água e, por conseguinte, pode-se atribuir aos solos mais básicos um caráter hidrófilo, como definido por Michel et al. (2001). Os resultados, como os obtidos por Albuquerque et al. (2005) em Latossolo alumínico, indicaram não haver maior disponibilidade de água e conteúdo de água volumétrica na superfície do solo sob plantio direto com a calagem.

No presente estudo, o Argissolo com correção da acidez do solo até $\mathrm{pH}$ 6,0 apresentou-se mais hidrófilo e com maior umidade gravimétrica ao final do ensaio de ascensão capilar que sob condição original de acidez (Quadro 1). Esses resultados concordam com observações de Pernes-Debuyser \& Tessier (2004) de que solos que receberam historicamente fertilizantes básicos apresentaram maiores velocidades de infiltração de água que solos mais ácidos. Nesse contexto, pode-se avançar na hipótese de que solos ácidos e básicos comportam-se de modo diferente em relação à água, apesar de a densidade do solo não ter sido sensível. Isso demonstra que a estrutura do solo recuperou-se após a incorporação do corretivo, ao passo que manteve boas características químicas ao longo de mais de uma década (Quadro 1). A afinidade da fração sólida pela água, traduzida aqui pela velocidade de umectação, e a retenção de água são alteradas pelo histórico de calagem do solo, enquanto, a densidade do solo não demonstrou estar ligada ao efeito da calagem sobre a eletroquímica do solo.

Estudos em laboratório são ainda necessários para avaliar o efeito isolado, nas propriedades físicas, da

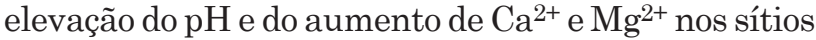
de troca decorrentes da calagem. No entanto, a calagem não pode ser restrita somente ao controle de algumas propriedades químicas do solo, mas, sim, considerada como uma prática com efeitos abrangentes, responsável por modificações nas características químicas, físicas e biológicas do solo, e inserida principalmente num contexto de sustentabilidade agrícola (Andreoli \& Tellarini, 2000; Virén-Lehr, 2001).

\section{CONCLUSÕES}

1) A calagem altera a relação entre solo e água, tornando o solo mais ávido à água, logo, mais hidrófilo, com o aumento das doses de calcário.

2) Após 12 anos da calagem, as formas de aplicação de calcário não tiveram influência sobre a densidade do solo e retenção de água.

\section{AGRADECIMENTOS}

Os autores agradecem a FAMV/UPF, pela liberação de recursos emergenciais para que o projeto chegasse a termo, e a FAPERGS, pela concessão de uma bolsa de iniciação científica.

\section{LITERATURA CITADA}

ALBUQUERQUE, J.A.; MAFRA, A.L.; FONTOURA, S.M.V.; BAYER, C. \& PASSOS, J.F.M. Avaliação de sistemas de preparo e calagem em um Latossolo Bruno alumínico. R. Bras. Ci. Solo, 29:963-975, 2005.

ANDREOLI, M. \& TELLARINI, V. Farm sustainability evaluation: Methodology and practice. Agric. Ecosyst. Environ., 77:43-52, 2000.

BORTOluzZI, E.C.; PERNES, M. \& TESSIER, D. Interestratificado caulinita-esmectita em um Argissolo desenvolvido a partir de rocha sedimentar do sul do Brasil. R. Bras. Ci. Solo, 31:1291-1300, 2007.

BORTOLUZZI, E.C.; RHEINHEIMER, D.S. \& TESSIER, D. An estimation of water retention properties in sandy soil of Southern Brazil. In: SYMPOSIUM INTERNATIONAL ON THE MANAGEMENT OF TROPICAL SANDY SOILS: A holistic approch for sustainable development of problem soils in the tropics, 1., Khon Kaen, 2005. Proceedings. Khon Kaen, FAO, 2005. p.189-193. 
BORTOLUZZI, E.C. \& TESSIER, D. La pratique du chaulage en système de semis direct: Une expérience au Sud du Brésil (Rio Grande do Sul). Étude Gestion Sol, 9:187-196, 2002.

BORTOLUZZI, E.C.; TESSIER, D.; RHEINHEIMER, D.S. \& JULIEN, J.L. The cation exchange capacity of a sandy soil $\mathrm{n}$ southern Brazil: an estimation of permanent charge and pH-dependent charges. Eur. J. Soil Sci., 57:356-364, 2006.

BORTOLUZZI, E.C.; VELDE, B.; PERNES, M.; DUR, J.C. \& TESSIER. D. Vermiculite with hydroxy-aluminium interlayer and kaolinite formation in a subtropical sandy soil from south Brazil. Clay Minerals, 43:155-163, 2008.

CAIRES, E.F.; BANZATTO, D.A. \& FONSECA, A.F. Calagem na superfície em sistema plantio direto. R. Bras. Ci. Solo, 24:161-169, 2000.

CHENU, C.; LE BISSONNAIS, Y. \& ARROUAYS, D. Organic matter influence on clay wetability and soil aggregate stability. Soil Sci. Soc. Am. J., 64:1479-1486, 2001.

COMISSÃO DE QUÍMICA E FERTILIDADE DE SOLO DO RIO GRANDE DO SUL E DE SANTA CATARINA CQFSRS/SC. Recomendações de adubação e de calagem para os Estados do RS e SC. 3.ed. Passo Fundo, SBCSNúcleo Regional Sul, 2004. 400p.

COSTA, F.S.; BAYER, C.; ALBUQUERQUE, J.A. \& FONTOURA, S.M.V. Calagem e as propriedades eletroquímicas e físicas de um Latossolo em plantio direto. Ci. Rural, 34:281-284, 2004.

EMPRESA BRASILEIRA DE PESQUISA AGROPECUÁRIA EMBRAPA. Sistema brasileiro de classificação de solos. 2.ed. Brasília, 2006. 306p.

GOEBEL, M-O.; BACHMANN, J.; WOCHE, S.K. \& FISCHER, W. Soil wettability, aggregate stability, and the decomposition of soil organic matter. Geoderma, 128:8093, 2005.

GRIEVE, I.C.; DAVIDSON, D.A. \& BRUNEAU, P.M.C. Effects of liming on void space and aggregation in an upland grassland soil. Geoderma, 125:39-48, 2005.

JOUANNY, C.; CHENU, C. \& CHASSIN, P. Détermination de la mouillabilité des constituants du sol à partir de mesures d'angles de contact: Rev. Bibliogr. Sci. Sol, 30:33-47, 1992.

KAMINSKI, J.; RHEINHEIMER, D.S.; GATIBONI, L.C.; BRUNETTO, G. \& SILVA, L.S. Eficiência da calagem superficial e incorporada precedendo o sistema plantio direto em um Argissolo sob pastagem natural. R. Bras. Ci. Solo, 29:573-580, 2005.
KAMINSKI, J.; RHEINHEIMER, D.S.; SANTOS, E.J.S.; GATIBONI, L.C. \& BORTOLUZZI, E.C. Resposta de culturas à aplicação de calcário em superfície ou incorporado ao solo em plantio direto. Ci. Rural, 30:605609,2000

MICHEL, J.C.; RIVIÈRE, L.M. \& BELON-FONTAINE, M.N. Measurement of the wettability of organic materials in relation the water content by capillarity rise method. Eur. J. Soil Sci., 459-467, 2001.

MONNIER, G.; STENGEL, P. \& FIES, J.C. Une méthode de mesure de la densité apparente de petits agglomérats terreux: Application à l'analyse des systèmes de porosité du sol. Ann. Agron., 24:533-545, 1973.

PELTIER, O. Raisonnement du chaulage des prairies: Influence sur la production et la qualité des fourrages. In: COLLOQUE L'ACIDIFICATION DES SOLS: ORIGINE, APPROCHE, ENJEUX ET MAÎTRISE. Versailles, AFES/ INRA, 2001. p.27-28

PERNES-DEBUYSER, A. \& TESSIER, D. Soil physic properties affected by long term fertilizer. Eur. J. Soil Sci., 55:505-512, 2004

PROST, R.; KOUTIT, T.; BENCHARA, A. \& HUARD, E. State and location of water adsorbed on clay minerals: Consequences of the hydration and swelling-shrinkage phenomena. Clays Clay Miner., 46:117-131, 1998.

RHEINHEIMER, D.S.; SANTOS, E.J.S.; KAMINSKI, J. \& XAVIER, F.M. Aplicação superficial de calcário no sistema plantio direto consolidado em solo arenoso. Ci. Rural, 30:263-268, 2000a.

RHEINHEIMER, D.S.; SANTOS, E.J.S.; KAMINSKI, J.; BORTOLUZZI, E.C. \& GATIBONI, L.C. Alteração de atributos do solo pela calagem superficial e incorporada a partir de pastagem natural. R. Bras. Ci. Solo, 24:797-805, $2000 \mathrm{~b}$

TEDESCO, M.J.; GIANELLO, C.; BISSANI, C.A.; BOHNEN, H. \& VOLKWEISS, S.J. Análise de solo, plantas e outros materiais. 2.ed. Porto Alegre, Universidade Federal do Rio Grande do Sul, 1995. 174p. (Boletim Técnico de Solos)

TESSIER, D. Influence des charges superficielles sur les propriétés physiques des sols. In: COLLOQUE L'ACIDIFICATION DES SOLS: ORIGINE, APPROCHE, ENJEUX ET MAITRISE. Versailles, AFES/INRA, 2001. p.13-14.

VIRÉN-LEHR, S.von. Sustainability in agriculture: An evaluations of principal goal-oriented concepts to close the gap between theory and practices. Agric. Ecosyst. Environ., 84:115-129, 2001. 